highly sensitive screening assay for abnormal disease-associated prion protein ( $\mathrm{PrPSc}^{\mathrm{Sc}}$.

$\mathrm{PrPSc}^{\mathrm{S}}$ is thought to replicate in vivo via conversion of normal host cellular prion protein $(\mathrm{PrP})$. PMCA rapidly reproduces this conversion process in vitro, thereby allowing detectable levels of PrPSc to be generated. Jones et al. showed that 48-cycle PMCA significantly amplified protease-resistant prion protein (PrPres; a marker of $\mathrm{PrP}^{\mathrm{Sc}}$ detectable by western blotting) after seeding of vCJD brain homogenate into both human and humanized transgenic mouse brain tissue substrates. Significant amplification was, however, observed only in brain tissue samples homozygous for methionine at codon 129 of the prion protein gene, PRNP. After PMCA, CDI had comparable detection sensitivity to western blotting, but the former technique has the theoretical advantage of enabling detection of proteinase-K-sensitive $\mathrm{PrPSc}$.

The authors also investigated the possibility of using human apheresis platelets, which express $\mathrm{PrPC}^{\mathrm{C}}$ on their surface, as an alternative substrate for PrPSc amplification that would obviate the requirement for human brain tissue. After PMCA, $\mathrm{PrPSc}^{\mathrm{S}}$ amplification was detectable by CDI in two of three platelet batches. These platelets had not, however, been approved for genetic analysis, and consequently the authors could only speculate that amplification success was dependent on PRNP codon 129 polymorphism.

Original article Jones M et al. (2007) In vitro amplification and detection of variant Creutzfeldt-Jakob disease $\mathrm{PrP}^{\mathrm{Sc}}$. J Pathol 213: 21-26

\section{Human neural stem cells improve function in primate model of severe Parkinson's disease}

In a recent paper, Redmond et al. report that transplantation of human neural stem cells (hNSCs) leads to functional improvement in a primate model of severe Parkinson's disease. Importantly, their study indicates that the stem cells seem to act not only by replacement of dopaminergic cells, but also by supporting multiple endogenous repair systems.

In the study, undifferentiated hNSCs were implanted into the right substantia nigra (SN) and the bilateral caudate nuclei of adult African green monkeys with severe Parkinson's disease. Four months after implantation, monkeys treated with hNSCs showed significant behavioral and functional improvements, measured by a Parkinson's factor score, compared with those that underwent a sham operation $(P<0.05)$. Histological examination demonstrated robust survival and migration of engrafted hNSCs, which differentiated into various neural stem cell types. A small number of graft-derived cells were found in the lesioned host SN, where they expressed markers consistent with a dopaminergic phenotype. Many other cells migrated along the nigrostriatal pathway, where they closely associated with host-derived cells, and differentiated into astrocytic cells expressing factors that protect dopaminergic cells. As well as preserving the host nigrostriatal circuitry, hNSC-derived cells were associated with a normalizing effect on the size and number of endogenous neurons in the $\mathrm{SN}$, as well as normalization of $\alpha$-synuclein aggregation.

The authors hypothesize that intrinsic signals in the lesioned host might direct differentiation of hNSCs to promote homeostasis of SN neurons and their nigrostriatal projections, thereby restoring function.

Original article Redmond DE Jr et al. (2007) Behavioral improvement in a primate Parkinson's model is associated with multiple homeostatic effects of human neural stem cells. Proc Natl Acad Sci USA 104: 12175-12180

\section{Newly designed drugs mimic effect of mood stabilizers}

The mechanism of action of mood stabilizing drugs such as lithium and valproic acid is unknown, but there is growing evidence that specific inhibitors of the enzyme glycogen synthase kinase- $3 \beta$ (GSK-3 $\beta$ ) could mimic the effects of these agents. Kozikowski et al. propose that structure-based design methods could be used to develop novel GSK-3 $\beta$ inhibitors to treat bipolar disorder and other neurodegenerative diseases, without the adverse effects associated with present treatments.

The investigators focused on benzofuranylindolyl-maleimides, a little-studied group of molecules that inhibit GSK-3 $\beta$. Modeling studies were used to identify specificity determinants in order to achieve more-specific inhibition of GSK-3 $\beta$; ligands were then designed to fit the catalytic site spatially, and to bind to amino acid residues unique to the binding site of GSK-3 $\beta$. The synthesized molecules were tested in vitro for their ability to inhibit GSK-3 $\beta$, 